\title{
The importance of initial conditions and metallicity for the fragmentation of protogalactic gas
}

\author{
Anne-Katharina Jappsen ${ }^{1}$, Simon C. O. Glover ${ }^{2}$, Mordecai-Mark Mac \\ Low $^{3}$ and Ralf S. Klessen ${ }^{2}$ \\ ${ }^{1}$ School of Physics and Astronomy, Cardiff University, Cardiff, CF24 3AA, UK \\ email: jappsena@cf.ac.uk \\ ${ }^{2}$ Institut für Theoretische Astrophysik, Universität Heidelberg, 69120 Heidelberg, Germany \\ ${ }^{3}$ American Museum of Natural History, New York, NY, 10024-5192, USA
}

\begin{abstract}
The formation of the first stars out of metal-free gas appears to result in stars at least an order of magnitude more massive than in the present-day case. We here consider what controls the transition from a primordial to a modern initial mass function. We study the influence of low levels of metal enrichment and different initial conditions on the cooling and collapse of initially ionized gas in small protogalactic halos using three-dimensional, smoothed particle hydrodynamics simulations. We argue that fragmentation at moderate density depends on the initial conditions for star formation more than on the metal abundances present.
\end{abstract}

Keywords. stars: formation, stars: mass function, early universe, hydrodynamics

In a seminal paper, Bromm et al. (2001) performed simulations of the collapse of cold gas in a top-hat potential that included the metallicity-dependent effects of atomic finestructure cooling. In the absence of molecular cooling, they found that fragmentation suggestive of a present-day initial mass function only set in at metallicities above a threshold value of $Z \simeq 10^{-3.5} Z_{\odot}$. However, they noted that the neglect of molecular cooling could be significant. Omukai et al. (2005) argued, based on the results of their detailed one-zone models, that molecular cooling would indeed dominate the cooling over many orders of magnitude in density.

The effects of molecular cooling at densities up to $n \approx 500 \mathrm{~cm}^{-3}$ were studied by Jappsen et al. (2007) in three-dimensional collapse simulations of warm ionized gas in minihalos for a wide range of environmental conditions. This study used a time-dependent chemical network running alongside the hydrodynamic evolution as described in Glover \& Jappsen (2007). The physical motivation was to investigate whether minihalos that formed within the relic HII regions left by neighboring Pop III stars could form subsequent generations of stars themselves, or whether the elevated temperatures and fractional ionizations found in these regions suppressed star formation until larger halos formed. In this study, it was found that molecular hydrogen dominated the cooling of the gas for abundances up to at least $10^{-2} Z_{\odot}$. In addition, there was no evidence for fragmentation at densities below $500 \mathrm{~cm}^{-3}$.

Jappsen et al. (2009b) investigated the behaviour of hot, initially ionized gas in a fixed DM potential, with varying values of rotation, turbulence and metallicity. These models showed no fragmentation during collapse to number densities as high as $10^{5} \mathrm{~cm}^{-3}$, for metallicities reaching as high as $10^{-1} Z_{\odot}$, far above the threshold suggested by previous work. Jappsen et al. (2009a) showed that gas in simulations with low initial temperature, moderate initial rotation, and a top-hat DM overdensity, will readily fragment into 

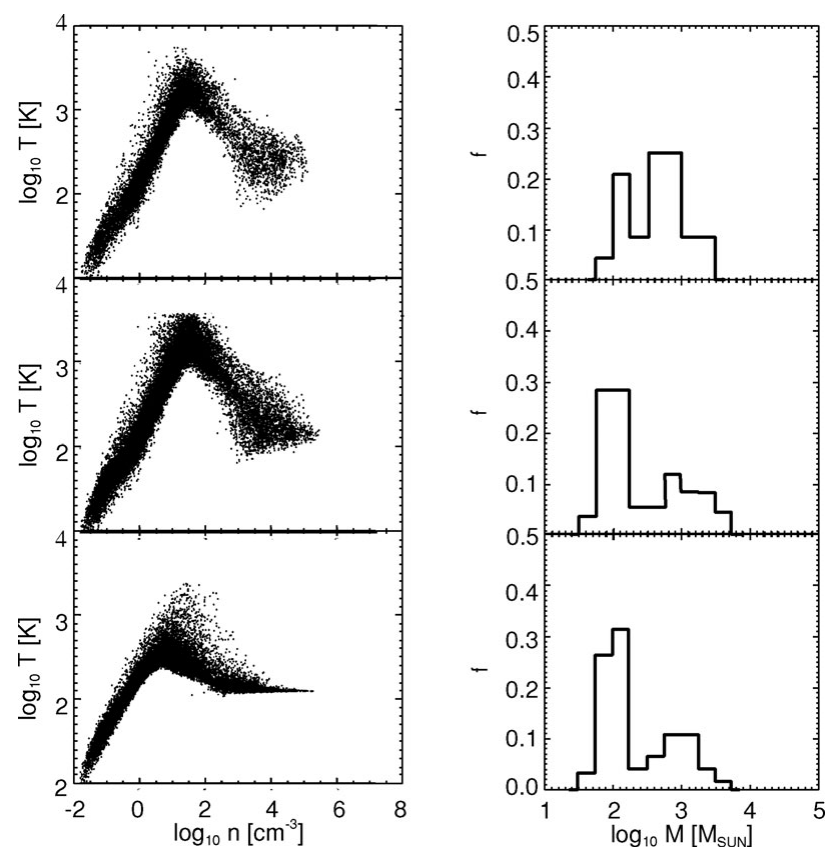

Figure 1. Gas temperature versus $H$ density (left-hand side) and mass distribution of clumps (right-hand side) for gas collapsing in a typical minihalo, shown for the primordial case and pre-enriched to $Z=10^{-3}$ and $Z=10^{-1} \mathrm{Z}_{\odot}$, from top to bottom (see also Jappsen et al. 2009a). The temperature evolution of primordial gas is very similar to the $Z=10^{-3} \mathrm{Z}_{\odot}$ case, showing that metal-line cooling becomes important only for very high metallicities. The fraction of lowmass fragments increases with higher metallicity, since more gas can cool to the temperature of the CMB before becoming Jeans-unstable. However, the fragments are still very massive, suggesting that metal-line cooling might not be responsible for the transition to low-mass stars.

multiple objects, regardless of metallicity, provided that enough $\mathrm{H}_{2}$ is present to cool the gas. Rotation leads to the build-up of massive disk-like structures in these simulations, which allow smaller-scale fluctuations to grow and become gravitationally unstable. The resulting mass spectrum of fragments peaks at a few hundred solar masses, roughly corresponding to the thermal Jeans mass in the disk-like structure (see Fig. 1).

These results suggest that the initial conditions adopted by Bromm et al. (2001) may have determined the result much more than might have been appreciated at the time. The actual initial conditions to be considered still need to be determined in detail by observation and modeling of galaxy formation. Metal abundance may still drive fragmentation at very high densities due to dust cooling, perhaps giving an alternative metallicity threshold (Clark et al. 2008).

\section{References}

Bromm, V., Ferrara, A., Coppi, P. S., \& Larson, R. B. 2001, MNRAS, 328, 969

Clark, P. C., Glover, S. C. O., \& Klessen, R. S. 2008, ApJ, 672, 757

Glover, S. C. O. \& Jappsen, A.-K. 2007, ApJ, 666, 1

Jappsen, A.-K., Glover, S. C. O., Klessen, R. S., \& Mac Low, M.-M. 2007, ApJ, 660, 1332

Jappsen, A.-K., Klessen, R. S., Glover, S. C. O., \& MacLow, M.-M. 2009a, ApJ, 696, 1065

Jappsen, A.-K., Mac Low, M.-M., Glover, S. C. O., Klessen, R. S., \& Kitsionas, S. 2009b, ApJ, 694,1161

Omukai, K., Tsuribe, T., Schneider, R., \& Ferrara, A. 2005, ApJ, 626, 627 